\title{
Views of Catholic Priests Regarding Causes, Treatments and Psychosocial Consequences of Schizophrenia and Depression: A Comparative Study in Italy
}

\author{
Lorenza Magliano $^{1}\left(\mathbb{1} \cdot\right.$ Giulia Citarelli $^{1}\left({ }^{\oplus} \cdot\right.$ Gaetana Affuso $^{1}(\mathbb{1}$
}

Accepted: 17 November 2020 / Published online: 2 December 2020

(C) The Author(s) 2020

\begin{abstract}
This study explored views of Catholic priests about schizophrenia and depression in Italy. Participants completed a questionnaire on their views about either schizophrenia $(N=282)$ or depression $(N=277)$. The depression group was surer than the schizophrenia group that: the disorder was due to psychosocial causes; curable; non-requiring long-term pharmacotherapy; the persons with depression could participate in religious activities. The older priests were more convinced than the younger priests that: the prayer and long-term pharmacotherapy are useful; the persons with mental disorders had affective difficulties, are recognizable and kept at distance. Priests should receive education on stigma in mental disorders, particularly schizophrenia.
\end{abstract}

Keywords Schizophrenia - Depression · Religion · Priests · Causal beliefs · Attitudes · Stigma

\section{Introduction}

Worldwide, around five billions of people adhere to a religion, mostly Christianity (2.2 billions) and Islam (1.6 billion; Hackett 2011). Like any member of society, some of these believers may suffer from a mental disorder (MD). Research reveals that persons with MDs mostly consider religion to be helpful in dealing with their own mental health problems (Koenig 2009; Mohr et al. 2012; Weber and Pargament 2014). In a US survey of 406 persons with long-term MDs, $80 \%$ of the sample used religion to cope with their symptoms (Tepper et al. 2001). In another US study of 157 persons with severe MDs, 50\% valued religious practice as useful for their mental status and as an alternative therapy to medical treatments (Russinova et al.

Lorenza Magliano

lorenza.magliano@unicampania.it

1 Department of Psychology, University of Campania "Luigi Vanvitelli”, Viale Ellittico 31, 81100 Caserta, Italy 
2002). In a study on 276 people with schizophrenia in Switzerland, Canada and North Carolina (USA), $87 \%$ of participants acknowledged the beneficial effects of religion on their mental health, while $13 \%$ stated that religion was distressing (Mohr et al. 2012). The relevance of religion from the perspective of persons with MDs is supported by studies showing that religious practice is associated with improvement of psychiatric symptoms. A systematic review of 43 studies carried out in the period 1990-2010 (Bonelli and Koenig 2013) found that religion/spirituality was associated with less severe psychiatric symptoms in $72 \%$ of cases. In particular, religious practice was found to be associated with less severe symptoms in $79 \%$ of studies on depression and $40 \%$ of studies on schizophrenia. Another study reported increased probability transient psychotic states associated with intense religious experiences (Linden et al. 2010). Adherence to religions among people with MDs is positively valued by mental health professionals (Bonelli and Koenig 2013; Vasegh et al. 2012) In a US study, $76 \%$ of participating psychiatrists acknowledged religion/spirituality as having beneficial effects on patients' mental state (Curlin et al. 2007). In another US study, $82 \%$ of participating psychologists thought religion more beneficial than harmful to mental health (Delaney et al. 2007).

In many religions, spiritual leaders also serve as advisors in non-religious matters, such as health problems. However, the role of spiritual leaders in these matters may differ according to religion. Priests are more likely to be contacted for mental health problems than psychiatrists and psychologists combined (Hohmann and Larson 1993). Compared to psychiatrists and psychologists, priests sometimes are perceived as more capable of warmth, caring, professionalism and listening skills (Milstein et al. 2003, cited by Ellison et al. 2006). In a study on persons with MDs (Wang et al. 2003), 25\% had sought a priest's advice and of these, $25 \%$ had a severe MD. Moreover, $23 \%$ of the sample had contacted priests before general practitioners, psychiatrists or other specialists and $56 \%$ had contacted only priests in the previous year. Another study exploring the ability of psychologists and rabbis to identify schizophrenia in a case-vignette setting found no significant difference in the diagnostic skills among these groups. Compared to psychologists, rabbis were more skeptical regarding the usefulness of psychotropic drugs, and they more tended to interpret schizophrenia as due to religious causes (Milstein et al. 2000). It should be also mentioned that some persons with MDs avoid seeking treatments from physicians and therapists and prefer to seek the help of priests instead. Sometimes this is because persons with MDs are not trusting of medical systems or medication or fearful of them (Carey and Del Medico 2013). The involvement of priests as consultants in mental health issues varies according to the type of MD. In a US study on 1231 lay people, $78.8 \%$ of respondents viewed clergy as helpers in the case of schizophrenia and $91.0 \%$ in the case of depression (Ellison et al. 2006).

Given the relevance of priests' advice for believers with MDs, it is worthwhile to investigate priests' beliefs regarding persons with these disorders. However, only a few studies have specifically examined this issue. In a survey among 115 members of the Catholic clergy in Lebanon (Aramouny et al. 2020), 85\% of the sample indicated traumatic experiences and 75\% named stress among the causes of MD. Chemical imbalance was reported by $70 \%$ of respondents, and heredity by $48 \%$ of them. Supernatural causes, such as diabolical possession, were reported by $22 \%$ of the 
sample. Twenty-six percent believed that prayer was more important than drugs as treatment of MDs, although $80 \%$ of the sample valued drugs as helpful to cope with daily stress. In this study, levels of authoritarianism were found to be higher among clergy members who were surer of the supernatural origin of MDs and lower among clergy members with previous contacts with persons with MDs. A study conducted in Sydney on the views regarding causes of MD and effects of drugs (Youssef and Deane 2003) among 170 Arabic-speaking clerics found that most of the sample viewed drug and alcohol addiction (93.5\%) and psychosocial factors (stress 93\%, trauma 91\%) as very/somewhat important causes of MD, while biological factors and religious causes were less frequently endorsed (heredity $76.5 \%$ and chemical imbalance $64.1 \%$; spiritual poverty $84.7 \%$ and possession $57.1 \%$ ). Prayer/spirituality was considered more useful than drugs by $43 \%$ of the sample.

Conversely, in a study of 1031 US Methodist pastors (Lafuze et al. 2002), biological causes were considered more important than psychosocial causes, religious causes and fate. Moreover, 53\% of the sample considered persons with MD more dangerous than the others. None of the above-mentioned studies has been carried out in Europe, where $72 \%$ of the population is Christian and $48 \%$ are Catholic (Eurobarometer 2012). Furthermore, most studies have explored clergy beliefs regarding MDs generically, which could make the results not fully representative of priests' beliefs about MDs with high stigma, such as schizophrenia. Finally, most studies concerned clergy views of causes and treatments of MDs, while almost no study specifically explored priests' attitudes about persons with MDs participating in religious celebrations and group activities.

In the period 2017-2019, we conducted a study on views regarding persons with MD in a large sample of Catholic priests in Italy, a country where $74 \%$ of people (about 45 millions) claims to be Catholic (Ipsos Game Changers 2017). Participants were invited to read either a random description of schizophrenia or depression and then to complete a questionnaire on "Persons with a condition like that reported in the description". Data were collected face-to-face in the parish churches, using a validated questionnaire (Magliano et al. 2017a, 2018) that was adapted to the survey context and re-validated. The views measured were about causal belief, recovery, usefulness of bio-psychological treatments and religious practices, the need for long-term drug treatments, insight, recognizability and affective difficulties of persons with MDs, dangerousness and social distance from persons with MDs, and persons with MDs participating in parish activities and religious ceremonies. In this paper, we report the priests' views on the variables mentioned above and test whether the priests' views varied in relation to the type of described disorder. We predicted that compared to priests who completed the questionnaire after reading a clinical description of depression, those who completed the same questionnaire after reading a clinical description of schizophrenia: would place more relevance on biological factors and less relevance on psychosocial factors in the development of the disorders, would give more relevance to biomedical treatments, have more negative attitudes toward persons with MDs, and would be more skeptical regarding the persons with schizophrenia participating in religious activities and celebrations. We also tested whether priests' views of persons with MDs would be influenced by the priest's age. We expected that compared to the younger priests, the older priests 
would give less relevance to psychosocial factors as causes of MDs; are more convinced of the need of long-term biomedical treatments; have more negative attitudes toward persons with MDs, and are more uncertain about the participation of persons with MDs in religious activities and celebrations.

\section{Methods}

\section{Study Design}

The study was approved by the Research Ethical Board of the Department of Psychology of the University of Campania "Luigi Vanvitelli" (n. 22/2016 Department Board 6/12/16, and 16/2019 Department Board 14/5/2019) and conducted in accordance with the Helsinki Declaration. Data were collected from February 2017 to December 2019. The study was carried out on Catholic priests in parishes included in six religious jurisdictions located in Southern Italy and serving 181 populated areas. Jurisdictions were selected opportunistically considering the geographical closeness to the Department of Psychology. Parishes were identified by referring to the official websites of religious jurisdictions. Parish priests were contacted personally or by phone by a researcher and invited to participate in a survey regarding their views about persons with MDs. Eligible participants were asked to consent to participate by providing them with detailed information about the purpose of the study and how the data would be collected and processed (anonymity, aggregated data analysis, right of the participant to stop completing the questionnaire at any time and leave the questionnaire blank). Informed consent was sought in writing. However, several priests preferred to participate by giving verbal consent. Participants were asked to complete the priest version of the Opinions on mental illness Questionnaire (OQ-priest) after reading a randomly chosen clinical description of either schizophrenia ("Appendix 1") or depression ("Appendix 2"). The questionnaire was selfadministered either in the presence of the researcher at the parish church or in his/ her absence, according to the participant's preference. Information on priests' demographic variables (age and level of education), professional background (duration in the priesthood; years lived in the jurisdiction; role in the parish church; commitment in other activities, such as social or teaching activities, in addition to ecclesiastical function) and experience with persons with MDs (knowledge of persons with MDs among churchgoers, friends, family and priests; reasons why persons with MDs may attend the parish) were also collected.

\section{Assessment Instrument}

The OQ-priest is a self-reported tool available in Italian. The tool includes items from the OQ and ad hoc added items to specifically investigate the study context. From the OQ previously validated questionnaire (Magliano et al. 2017a, 2018), items of the following sections were used: (a) causal beliefs section: sixteen yes/ no items grouped into four factors: biological, substance-abuse, stress-related 
and traumatic causes of MD; (b) recommended professionals section: four yes/no items on health professionals to be involved in the treatment of persons with MD; (c) psychosocial consequences section: seventeen items grouped into the following factors: possibility of recovery, the usefulness of pharmacological and psychological therapies, need for long-term pharmacological therapies, the insight of persons with MDs, perception of social distance from persons with MDs and dangerousness, difficulties of persons with MDs in having romantic relationships. Section c items were rated on a 3-point scale from $1=$ "not true" to $3=$ "completely true". Furthermore, four causes were added to section $a$ (wrong therapy, frequenting bad company, possession, and magic), and two items to section $b$ (priest and exorcist as recommended professionals). Moreover, 12 items were added to section $c$ that were potentially groupable into five subscales: recognizability of persons with MDs, usefulness of prayer and exorcism as therapies for MDs, parenting skills of persons with MDs, opportunity for persons with MDs to be involved in sacraments and parish activities, and behaviors to be adopted with these persons during religious celebrations (same rating scale as for OQ section $c$ items). In this study, the psychometric properties of the OQ-priest version have been tested using confirmatory factor analysis (CFA) and Cronbach's $\alpha$ coefficient (see Statistical Analyses, Results sections and Tables 1, 2).

Table 1 Priest sociodemographic variables, professional characteristics and contact with persons with MDs (total sample, $N=559$ )

\begin{tabular}{ll}
\hline Age, mean \pm SD & $52.7 \pm 13.1$ \\
Education, $N(\%)$ & \\
Secondary school degree & $6(1.1)$ \\
Bachelor degree & $450(80.5)$ \\
Master's degree or Ph.D. in Theology & $103(18.4)$ \\
Years lived in the jurisdiction, mean \pm SD & $30.5 \pm 21.3$ \\
$\begin{array}{l}\text { Duration in the priesthood, mean } \pm \text { SD } \\
\text { Role in the parish church, } N(\%)\end{array}$ & $22.6 \pm 14.4$ \\
$\begin{array}{l}\text { Parish priest } \\
\text { Auxiliary parish priest }\end{array}$ & $463(82.8)$ \\
Knowledge of persons with MDs attending the parish \\
church, yes, $N$ (\%) & $96(17.2)$ \\
Reasons that persons with MDs attended the parish & $519(92.8)$ \\
church, $N(\%)$ & \\
Attending celebrations & \\
Participating in religious group & \\
Participation in voluntary activities & $313(57.7)$ \\
Receiving individual support & $156(28.8)$ \\
Receiving economic help & $89(16.4)$ \\
Receiving advices & $301(55.5)$ \\
Attending rituals aimed to solve the MD & $168(31.0)$ \\
Other reasons & $285(52.6)$ \\
\hline & $39(7.2)$ \\
& $26(7.2)$ \\
\hline
\end{tabular}


Table 2 Priests' views of factors involved in the development of schizophrenia and depression (total sample, $N=558$ )

\begin{tabular}{lcc}
\hline Causes & $N$ & $(\%)$ \\
\hline Traumatic events & 312 & 55.9 \\
Family conflicts & 291 & 52.2 \\
Stress & 285 & 51.1 \\
Psychological violence & 253 & 45.3 \\
Bereavements & 246 & 44.1 \\
Disillusionment in love & 232 & 41.6 \\
Use of street drugs & 211 & 37.8 \\
Work difficulties & 209 & 37.5 \\
Poor parenthood & 182 & 32.6 \\
Sexual abuses & 181 & 32.4 \\
Use of alcohol & 179 & 32.1 \\
Physical violence & 172 & 30.8 \\
Heredity & 165 & 29.6 \\
Physical illness & 135 & 24.2 \\
Magic & 101 & 18.1 \\
Frequenting bad company & 87 & 15.6 \\
Illness in pregnancy & 73 & 13.1 \\
Wrong therapy & 68 & 12.2 \\
Chemical imbalance & 64 & 11.5 \\
Possession & 57 & 10.2 \\
\hline One case missing & &
\end{tabular}

One case missing for causes' section

\section{Statistical Analyses}

CFA was performed on the global sample to verify the construct validity of the OQ-priest version, section $a$ items and section $c$ items, respectively. Maximum likelihood estimation of the covariances was applied. Cronbach's $\alpha$ values were computed to explore the content validity of the confirmed factors in each CFA. $\chi^{2}$ was used to compare the two groups (schizophrenia and depression) in their views about professionals to be involved in the care of persons with MD (OQ section $b$ ). The median of the priests' age was used to split the total sample into a group of younger priests and a group of older priests. Multivariate analysis of variance (MANOVA) was performed to compare the mean scores of causal factors (OQ section $a$ modified-dependent variables) among the two diagnostic groups (schizophrenia and depression-independent variables) and the two priest age groups (younger priest group and older priest group-independent variables). The same analysis was performed to compare the mean scores of psychosocial consequences factors (OQ section $c$ modified-dependent variables) among the two diagnostic groups (schizophrenia and depression-independent variables) and the two priest age groups (younger priest group and older priest group-independent variables). In both MANOVAs, interactions between the independent variables were also examined. The statistical significance level was set at $p<0.05$. CFAs were performed using the LISREL 8 Program (Jöreskog and 
Sörbom 1996). Other tests were performed using the SPSS package, version 21 (IBM 2012).

\section{Results}

\section{Participant Characteristics}

Of the 609 priests approached, 559 (91.8\%) agreed to participate and completed the questionnaire (reasons for non-participation: lack of time and/or not interested, 33; disagreement with research aims, 4; no opinion regarding persons with MD, 2; unknown, 5; unwilling to give such information to people outside the religious organization, 6). The socio-demographic and professional characteristics of the 559 participating priests are reported in Table 1. Participants had a mean age and a median age of $52.7( \pm 13.1$ SD) and 51.0 years, respectively. Most priests $(80.5 \%)$ had a bachelor's degree in theology. The 559 priests served 173 out of the 181 (95.6\%) inhabited areas included in the selected religious jurisdictions. Participants had a mean duration in the priesthood of 22.6 \pm 14.4 years and had lived on the jurisdiction area for 30.5 \pm 21.3 years. Among them, 82.8\% (463) were parish priest and $17.2 \%(N=96)$ were auxiliary parish priests. Nine-three percent of priests reported that persons with MDs attended the parish church, for reasons such as: participating in celebrations $(57.7 \%)$, religious groups $(28.8 \%)$ and voluntary activities (16.4\%); and, receiving individual spiritual support (55.5\%), advices (52.6\%), and economic help (31\%). Of the 559 respondents, 282 completed the OQ-priest after reading a clinical description of schizophrenia and 277 completed the same questionnaire after reading a clinical description of depression. Com'era prima: The two groups were comparable in all variables listed above except for the priests' contact with persons with MDs, which was higher in the depression group than in the schizophrenia group $\left(90.1 \%\right.$ vs. $96.8 \%, \chi^{2} 10.01$, $\left.d f 1, p<0.001\right)$. Furthermore, the number of respondents stating that persons with MDs attended the parish church to receive spiritual help and advices $\left(48.4 \%\right.$ vs. $62.9 \%, \chi^{2} 11.6, d f 1, p<0.001 ; 45.8 \%$ vs. $59.6 \%, \chi^{2} 10.2$, df $\left.1, p<0.001\right)$ and to attend religious groups and voluntary activities $\left(21.1 \%\right.$ vs. $36.7 \%, \chi^{2} 16.1, d f 1, p<0.0001 ; 10.2 \%$ vs. $22.8 \%, \chi^{2} 15.8, d f 1$, $p<0.0001$ ) was higher in the depression group.

\section{Descriptive Results of the Total Sample}

In the total sample of 558 ( 1 missing in causal beliefs section) priests, the factors most frequently reported as involved in the development of the MD were traumatic events $(55.9 \%)$, family conflicts $(52.2 \%)$ and stress $(51.1 \%$; Table 2$)$. Heredity was reported by $29,6 \%$ and chemical imbalance by $11.5 \%$ of the respondents. Magic and evil possession were reported by $18.1 \%$ and $10.2 \%$ of the priests, respectively. Seventy percent of priests recommended a psychologist and $52.9 \%$ a psychiatrist among the treating professionals. Moreover, $26.9 \%$ recommended a neurologist and $12.5 \%$ 
a general practitioner. Furthermore, $44.1 \%$ suggested the involvement of a priest and $7.2 \%$ an exorcist.

In the total sample of 559 priests, $57.7 \%$ of respondents thought it was "completely true" that persons with MD could recover, and $10.7 \%$ were equally sure that people with MD should take drugs for life (Table 3). Fifty-seven percent of respondents were convinced that psychological interventions are useful for the treatment of MD and $45.2 \%$ were sure of the usefulness of prayer. Forty-five percent of

Table 3 Priests' views of persons with schizophrenia and depression (total sample, $N=559$ )

\begin{tabular}{|c|c|c|c|c|c|c|}
\hline \multirow[t]{2}{*}{ Items } & \multicolumn{2}{|c|}{ Not true } & \multicolumn{2}{|c|}{$\begin{array}{l}\text { Partially } \\
\text { true }\end{array}$} & \multicolumn{2}{|c|}{$\begin{array}{l}\text { Com- } \\
\text { pletely } \\
\text { true }\end{array}$} \\
\hline & $N$ & $(\%)$ & $N$ & $(\%)$ & $N$ & $(\%)$ \\
\hline$\S$ Can recover & 26 & 5.0 & 196 & 37.3 & 303 & 57.7 \\
\hline Drugs are useful for $\S$ & 40 & 7.7 & 320 & 61.7 & 159 & 30.6 \\
\hline Psychological interventions are useful for $\S$ & 10 & 1.8 & 223 & 40.9 & 312 & 57.2 \\
\hline Prayer is useful for $\S$ & 31 & 5.7 & 266 & 49.1 & 245 & 45.2 \\
\hline Exorcism is useful for $\S$ & 242 & 52.4 & 178 & 38.5 & 42 & 9.1 \\
\hline$\S$ Must take drugs over the life & 246 & 54.8 & 155 & 34.5 & 48 & 10.7 \\
\hline If stop taking drugs, $\S$ become dangerous & 156 & 34.6 & 221 & 49.0 & 74 & 16.4 \\
\hline If stop taking drugs, $\S$ become unwell again & 87 & 19.1 & 251 & 55.2 & 117 & 25.7 \\
\hline$\S$ Are unpredictable & 74 & 14.5 & 281 & 55.1 & 155 & 30.4 \\
\hline$\S$ Are kept at distance by the others & 108 & 19.9 & 275 & 50.6 & 161 & 29.6 \\
\hline People does not know how to behave with $\S$ & 46 & 8.5 & 253 & 46.7 & 243 & 44.8 \\
\hline People does not understand the difficulties experienced by $\S$ & 47 & 8.7 & 262 & 48.3 & 233 & 43.0 \\
\hline People is frightened by $\S$ & 97 & 18.3 & 266 & 50.1 & 168 & 31.6 \\
\hline$\S$ Are dangerous to themselves & 63 & 12.4 & 343 & 67.5 & 102 & 20.1 \\
\hline$\S$ Are dangerous to others & 129 & 25.5 & 312 & 61.8 & 64 & 12.7 \\
\hline It is difficult for $\S$ to have a love relationship & 192 & 38.5 & 210 & 42.1 & 97 & 19.4 \\
\hline It is difficult for $\S$ to get married or to live together with a partner & 179 & 35.0 & 200 & 39.1 & 132 & 25.8 \\
\hline $\begin{array}{l}\S \text { Who are now well, may be responsible for parish activities (e.g., } \\
\text { catechesis, etc.) }\end{array}$ & 46 & 8.8 & 204 & 39.0 & 273 & 52.2 \\
\hline$\S$ Are reliable when they confess & 52 & 10.7 & 290 & 59.7 & 144 & 29.6 \\
\hline$\S$ Can be witnesses in the sacraments (e.g., marriage) & 66 & 12.7 & 162 & 31.2 & 292 & 56.2 \\
\hline$\S$ Can receive the Eucharist & 17 & 3.1 & 78 & 14.1 & 459 & 82.9 \\
\hline $\begin{array}{l}\text { During religious celebrations, } \S \text { create discomfort to other church- } \\
\text { goers }\end{array}$ & 278 & 52.2 & 219 & 41.1 & 36 & 6.8 \\
\hline $\begin{array}{l}\text { During religious celebrations, } \S \text { should be separated from other } \\
\text { churchgoers }\end{array}$ & 457 & 83.2 & 68 & 12.4 & 24 & 4.4 \\
\hline During religious celebrations, $\S$ should be supervised & 318 & 58.1 & 194 & 35.5 & 35 & 6.4 \\
\hline$\S$ Are recognizable & 142 & 27.0 & 257 & 49.0 & 126 & 24.0 \\
\hline$\S$ Do not realize that they are ill & 57 & 10.9 & 308 & 58.9 & 158 & 30.2 \\
\hline$\S$ Do not realize when they become unwell & 67 & 13.1 & 263 & 51.3 & 183 & 35.7 \\
\hline
\end{tabular}

$\S$ Persons with a condition like that reported in the clinical description 
priests thought that people does not know how to behave with persons with MD, while $29.3 \%$ thought that these persons are kept at a distance by the others. Thirty percent of participants were completely sure that these persons are unpredictable, while $12.7 \%$ of priests were completely sure that persons with MD are dangerous to others. Fifty-six percent fully agreed that persons with MD could be witness in the sacraments, and $52.2 \%$ fully agreed that these persons who have recovered could be responsible for parish activities. As far as the behaviors toward people with MD to be adopted during celebrations, $4.4 \%$ of priest firmly believed that these persons should be separated from other churchgoers and $6.4 \%$ firmly believed that persons with MD should be supervised.

\section{Confirmatory Factor Analyses (CFA)}

CFA performed on the 20 causes included in section $a$ of the questionnaire, confirmed a five-factor structure. The final model fits the data well, $\chi^{2}(558)=341.86$, df 160, $p<0.001$; non-normed fit index $[\mathrm{NNFI}]=0.93$; comparative fit index $[\mathrm{CFI}]=0.94$; root mean square error of approximation $[\mathrm{RMSEA}]=0.04,90 \% \mathrm{CI}$ $(0.04 ; 0.05)$; standardized root mean square residual $[\mathrm{SRMR}]=0.05$. All factor loadings were significant at the $p<0.001$ level. Cronbach's $\alpha$ values ranged from 0.48 to 0.75 . The details of the psychometric analyses for section $a$ are presented in Table 4 .

CFA performed on the 29 items included in section $c$ of the OQ-priest version confirmed the 13-factor structure listed above, with the exclusion of one item. The final model fits the data well, $\chi^{2}(559)=559.13$, df $252 p<0.05$; non-normed fit index $[\mathrm{NNFI}]=0.91$; comparative fit index $[\mathrm{CFI}]=0.93$; root mean square error of approximation [RMSEA] $=0.05,90 \%$ CI $(0.04 ; 0.05)$; standardized root mean square residual $[\mathrm{SRMR}]=0.05$. All factor loadings were significant at the $p<0.001$ level. Cronbach's $\alpha$ values ranged from 0.52 to 0.84 . The details of the psychometric analyses for section $c$ are presented in Table 5.

\section{Differences in Priests' Views Between the Two Groups}

MANOVA performed on causal factors 1-5 of the OQ-priest section $a$ revealed a significant effect of the diagnostic group on two of the factors (Wilks' $\lambda=0.93, F$ $(5,550)=7.88, p<0.0001$; Table 6). Otherwise, neither the priests' age (Wilks' $\lambda=0.98, F(5,550)=2.16, p<0.05)$ nor the interaction between the diagnostic group and the priests' age had any effect on the causal factors (Wilks' $\lambda=0.98, F$ $(5,550)=2.36, p<0.05$; no factor reached minimum level of accepted significance). In particular, MANOVA showed that in the schizophrenia group, priests reported less traumatic factors and stress-related factors among the causes of the disorder compared to the depression group. Regarding the professionals to be involved in the care of MD, in the schizophrenia group, a higher percentage of respondents recommended psychiatrists $\left(59.2 \%\right.$ vs. $\left.46.4 \%, \chi^{2}=9.2, d f 1, p<0.002\right)$ and not recommended psychologists $\left(65.2 \%\right.$ vs. $\left.75.4 \%, \chi^{2}=6.8, d f 1, p<0.009\right)$ and priests $(37.9 \%$ vs. $\left.50.4 \%, \chi^{2}=8.7, d f 1, p<0.003\right)$ among the treating professionals. 
Table 4 Confirmatory factor analysis on the QO section a items $(N=558)$

\begin{tabular}{|c|c|c|c|c|c|}
\hline \multirow[t]{2}{*}{ Items } & \multicolumn{5}{|c|}{ Factor loadings } \\
\hline & 1 & 2 & 3 & 4 & 5 \\
\hline Physical violence & 0.31 & & & & \\
\hline Psychological violence & 0.26 & & & & \\
\hline Traumatic events & 0.18 & & & & \\
\hline Sexual abuses & 0.31 & & & & \\
\hline Poor parenthood & 0.21 & & & & \\
\hline Heredity & & 0.10 & & & \\
\hline Chemical imbalance & & 0.13 & & & \\
\hline Illness in pregnancy & & 0.14 & & & \\
\hline Physical illness & & 0.18 & & & \\
\hline Wrong therapy & & 0.18 & & & \\
\hline Use of alcohol & & & 0.38 & & \\
\hline Use of street drugs & & & 0.36 & & \\
\hline Possession & & & & 0.15 & \\
\hline Frequenting bad company & & & & 0.19 & \\
\hline Magic & & & & 0.22 & \\
\hline Family conflicts & & & & & 0.20 \\
\hline Work difficulties & & & & & 0.26 \\
\hline Stress & & & & & 0.27 \\
\hline Bereavements & & & & & 0.25 \\
\hline Disillusionment in love & & & & & 0.26 \\
\hline Cronbach's $\alpha$ & 0.65 & 0.48 & 0.75 & 0.54 & 0.63 \\
\hline
\end{tabular}

Factor 1: traumatic causes; 2: biogenetic causes; 3: use of alcohol and street drugs; 4: supernatural causes; 5: stress-related causes

The correlations among the five factors were all significant for $p<0.01$ (standardized solution)

MANOVA performed on factors 1-13 of the OQ-priest section $c$ revealed a significant effect of the diagnostic group on five of the factors (Wilks' $\lambda=0.95, F$ $(13,543)=1.99, p<0.02$; Table 7$)$ and of the priests' age on five factors (Wilks' $\lambda=0.91, F(13,547)=3.97, p<0.0001)$. No significant interaction between the two independent variables was found (Wilks' $\lambda=0.98, F(13,547)=0.98, p>0.05$ ). In particular, MANOVA highlighted that in the schizophrenia group, priests were less optimistic about the possibility of recovery compared to the depression group. In the schizophrenia group, priests were surer of the need for long-term pharmacological treatments and more skeptical about the usefulness of prayer in the care of the disorder. Furthermore, in the schizophrenia group, priests were more convinced of the difficulties experienced by persons with MD in affective relationships and more doubtful regarding these persons participating of in parish activities and sacraments. As far as the effect of priests' age on priests' opinions, compared to the younger priests, the older priests acknowledged more relevance to prayer in the care of MDs and they were more convinced of the need for long-term pharmacological 


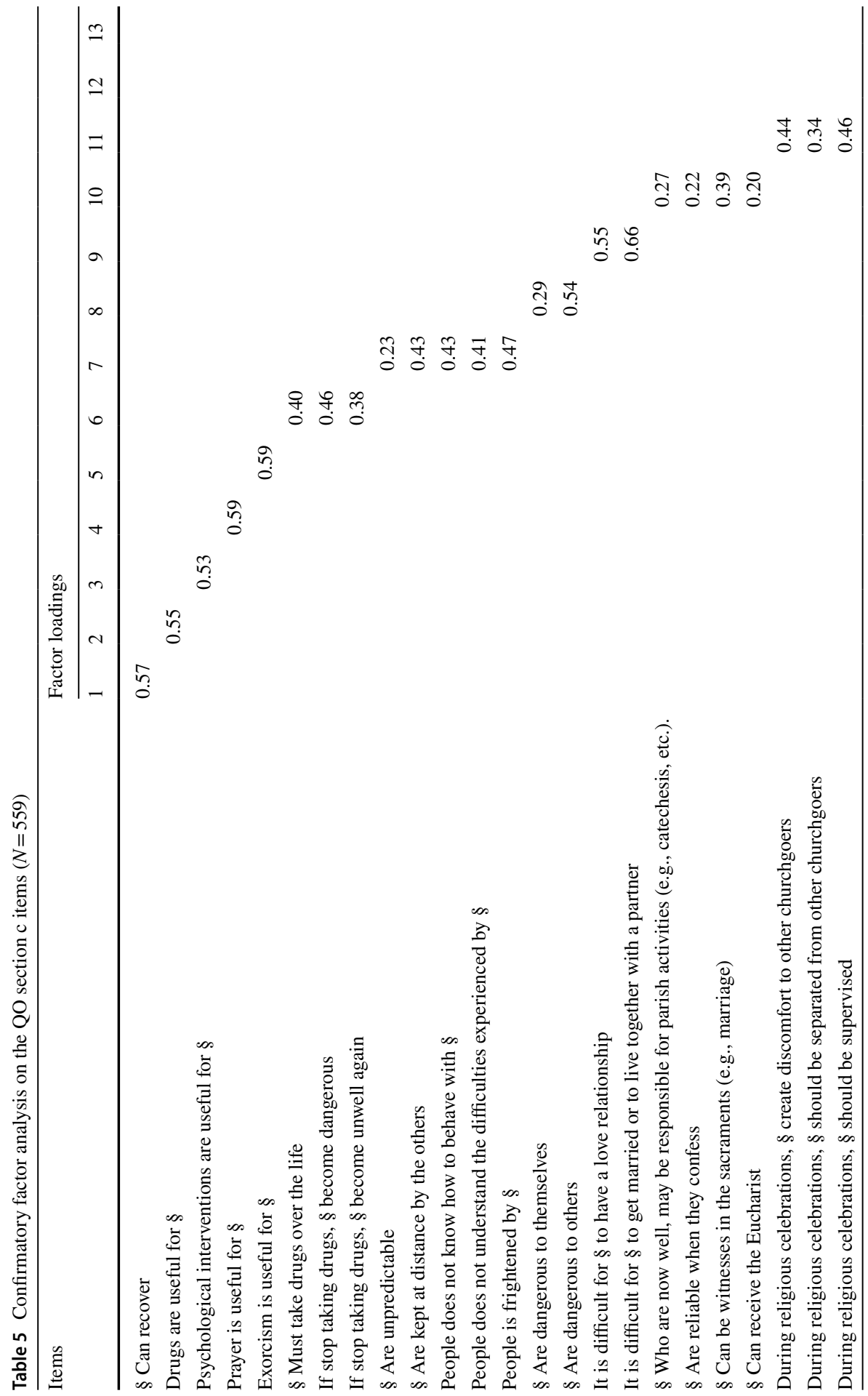




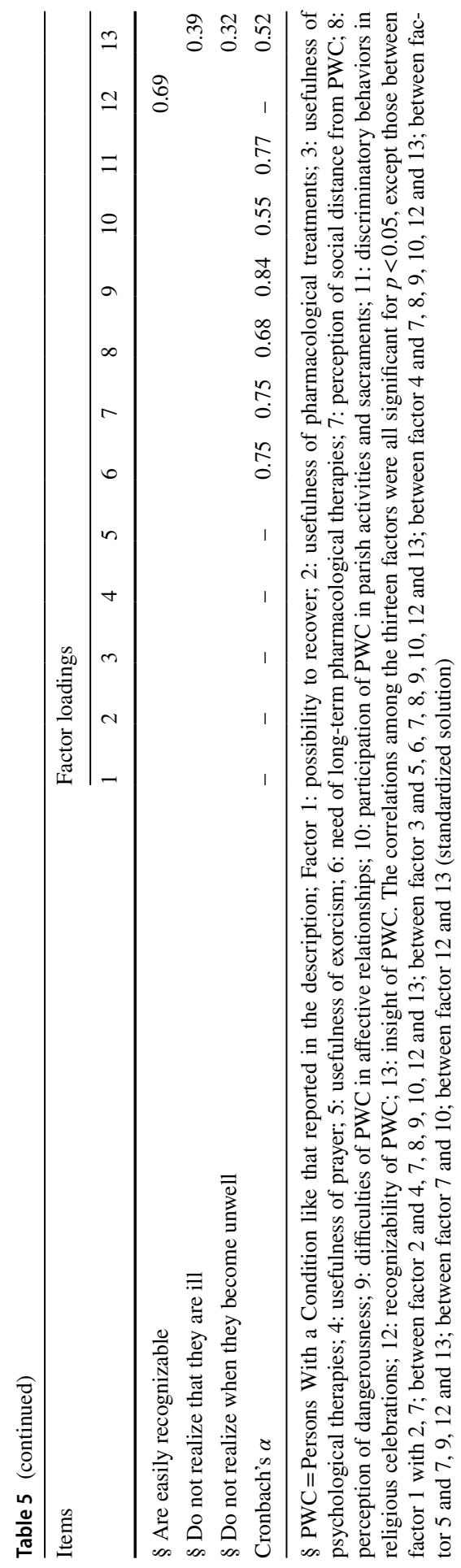


Table 6 Priests' views on causes of MDs: differences between diagnostic groups

\begin{tabular}{|c|c|c|c|}
\hline \multirow[t]{3}{*}{ Casual factors* } & \multicolumn{2}{|l|}{ Diagnostic groups } & \multirow{3}{*}{$\begin{array}{l}\text { MANOVA } \\
F(1,557)\end{array}$} \\
\hline & Schizophrenia $(N=281)$ & Depression $(N=277)$ & \\
\hline & Mean \pm SD & Mean \pm SD & \\
\hline Traumatic & $0.35 \pm 0.29$ & $0.44 \pm 0.32$ & $9.80^{\mathrm{a}}$ \\
\hline Stress-related & $0.37 \pm 0.30$ & $0.53 \pm 0.31$ & $35.9^{\mathrm{b}}$ \\
\hline Biogenetic & $0.17 \pm 0.21$ & $0.19 \pm 0.22$ & 0.35 \\
\hline Substances' misuse & $0.33 \pm 0.42$ & $0.37 \pm 0.43$ & 1.20 \\
\hline Supernatural & $0.13 \pm 0.24$ & $0.16 \pm 0.43$ & 1.65 \\
\hline
\end{tabular}

${ }^{\mathrm{a}} p<0.005 ;{ }^{\mathrm{b}} p<0.0001$

treatments. Moreover, the older priests were surer that persons with MDs had difficulties in affective relationships, were recognizable and were kept at distance by the others.

\section{Discussion}

\section{Interpretation of the Results}

The results of this study show that priests have both similarities and differences in their views of schizophrenia and depression and their attitudes toward persons with these disorders. In both diagnostic groups, priests attributed the disorder mainly to psychosocial causes and gave the lowest importance to supernatural causes. These data are in line with those from studies among Catholic clerics in Lebanon (Aramouny et al. 2020), Muslims and Catholics clergy in Ethiopia (Jacobsson and Merdasa 1991) and among Arabic-speaking religious leaders in Australia (Youssef and Deane 2003). Conversely, these findings are in contrast with the results of other studies carried out among American Methodist pastors (Lafuze et al. 2002), and among Euro-American Presbyterian pastors (Yamada et al. 2018).

The high importance given to psychosocial causes within each diagnostic group is probably due to the priests' high frequency of contact with people with MDs who attended the parish church with spiritual and material needs. As outlined by Aramouny et al. (2020), priests might be mostly exposed to persons whose mental health problems have been caused or strongly associated with traumatic events and problematic living conditions. In contrast with the stereotypical expectation of the priests as giving a prevalent religious interpretation of MDs, the mean score of the supernatural causes factor was low in both the groups. These results suggest that most priests tend to distinguish mental health problems from circumstances interpreted from a faith perspective (Leavey 2010). The low importance given to supernatural causes, a finding in line with that from the study by Aramouny et al. (2020), is also supported by the low percentage of priests indicating the exorcist among the recommended professionals in both the groups. These results might be partially 


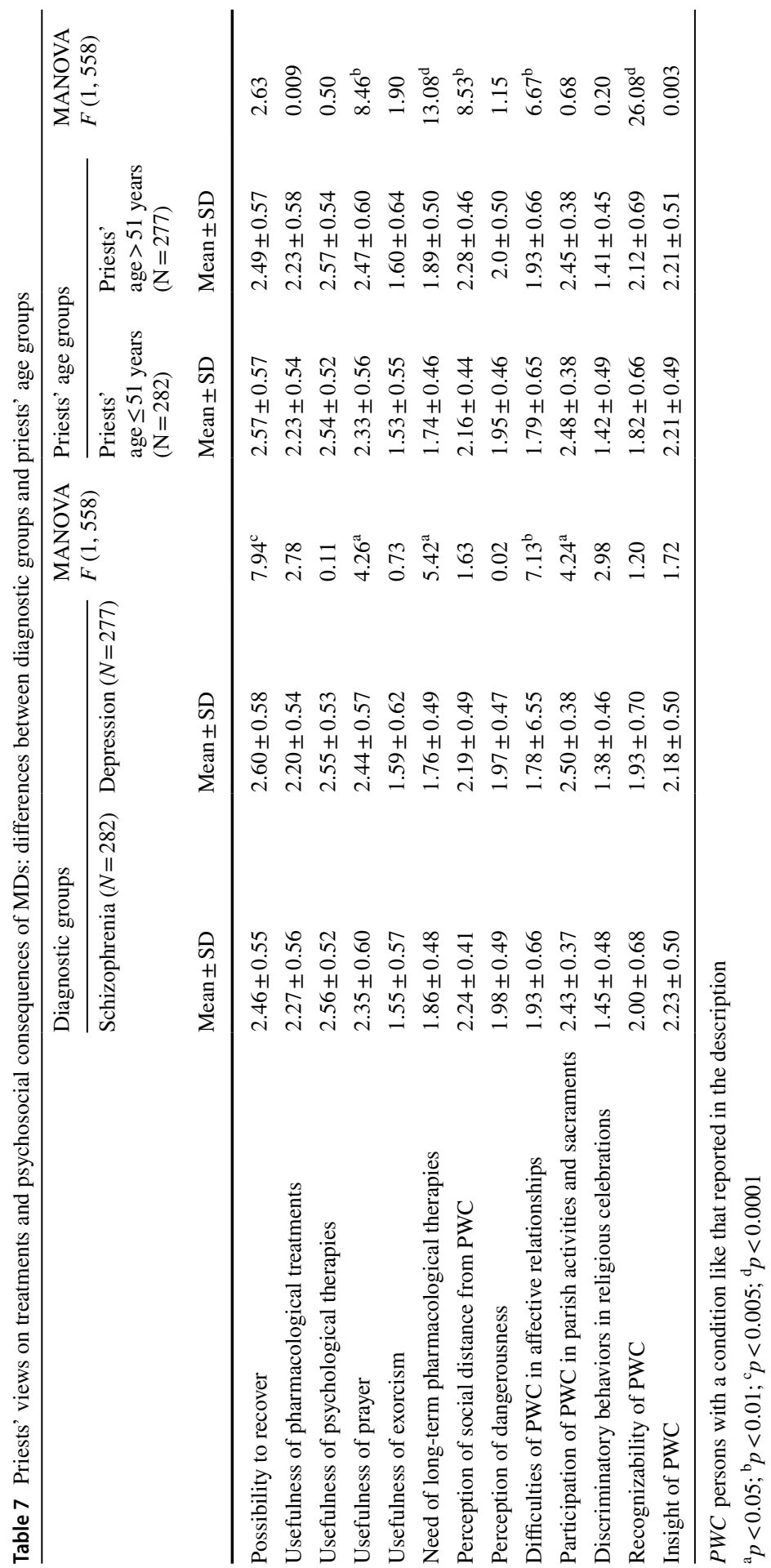


explained by a shift from Biblical times-when talk of demons and mental torment was not uncommon-to a bold medicalization of mental health problems, particularly schizophrenia, fostered by drug companies (Mosher et al. 2013) and the media (Magliano and Marassi 2018).

While the two diagnostic groups were similar in the relevance given to substance, biological and supernatural causes in the development of the disorder, the groups appear significantly different in the mean score of the stress-related and traumatic causal factors. The lower importance given to psychosocial causes in schizophrenia versus depression may be due to the lower frequency of priests having contact with persons with psychotic symptoms. Sometimes persons diagnosed with schizophrenia tend to exclude themselves in the belief that they are discriminated against (Thornicroft et al. 2009). Another reason for the difference in the relevance of psychosocial causal factors between the two diagnostic groups could be the different social representations of depression and schizophrenia in the media. Depression is frequently presented as due to a wide range of life adversities that can happen to everyone (Magliano et al. 2017b), whereas schizophrenia is frequently presented as a brain disorder that can be exacerbated by the stress (Magliano and Marassi 2018).

As far as the professionals to be involved in the care of MDs, psychologists and priests were more frequently recommended in the depression group, while psychiatrists were more frequently recommended in the schizophrenia group. The greater importance given to traumatic events and stress-related causes in depression can partly explain the high percentage of priests who indicated non-medical professionals for the treatment of this disorder. Beliefs about severity and need for longterm pharmacological interventions in schizophrenia may be a reason for the more frequent recommendation of the expertise of psychiatrists in this diagnostic group (Ellison et al. 2006). It should be highlighted that in nearly all cases, priests recommended religious professionals were in association with health professionals. This finding further confirms that most priests distinguish mental health problems from religious circumstances and view themselves and health professionals as meeting two different needs of believers with MD. However, it should not be excluded that the not very high percentage of priests believing in the healing power of God in schizophrenia and depression is related to the medicalization of MDs (Deacon 2013). As priests stated verbally, their involvement in the treatment of believers with MD should be intended as a source of spiritual support distinct from the health treatments. Priests should be trained on how to approach people with MDs and facilitate trusted relationships between these people and health care professionals, when necessary (Carey and Del Medico 2013). Data of this study suggest that collaboration between priests and mental health professionals is possible, while respecting each other's competence (Farrell and Goebert 2008).

Regarding the treatments of MD, in line with data from previous studies (Aramouny et al. 2020; Youssef and Deane 2003), priests value psychosocial interventions as more useful than pharmacological treatments. Moreover, no difference was found in the usefulness acknowledged for health treatments in schizophrenia and depression. This result may be important for the advices about therapies that priests give to believers with MDs. This is even more relevant in the case of schizophrenia, where treatments are unbalanced in favor of drug treatments, and low importance 
is given to evidence-based psychological treatments (Ince et al. 2016). The relevance given to mental health professionals and psychological therapies may have been influenced by an interview given by the Pope Francis to a French sociologist in 2017. In the interview that had great media resonance in Italy, the Pope referred to his personal experience of psychotherapy stating "I, too, who am Pope, needed help. I searched for it and benefited from it. Trust me. Get help" (cited by Carpiniello 2017). Priests are more skeptical regarding recovery in schizophrenia than in depression. The greater prognostic optimism regarding depression may be related to greater social acceptance of this disorder sometimes viewed as a transient experience that can affect everyone over the course of life (Munizza et al. 2013; Magliano et al. 2017b). Conversely, schizophrenia is often presented as an incurable brain disease requiring life-long pharmacological treatments to be controlled (Magliano and Marassi 2018b; Read et al. 2013; Kvaale et al. 2013). Skepticism about the possibility of recovery in schizophrenia can adversely affect the hope priests may convey to churchgoers with psychotic symptoms (Heffernan et al. 2016) and it may discourage persons with these disorders from making efforts to improve their quality of life.

We found no difference in the level of perceived dangerousness and social distance from persons with MD between schizophrenia and depression. However, it is not to be excluded that priests' responses to the items of these subscales would have been influenced by social desirability bias. As reported by Latkin et al. (2017), "social desirability bias is the tendency to underreport socially undesirable attitudes and behaviors and to over report more desirable attributes". Sometimes, socially desirable response might be explained by conscious and unconscious desires to avoid embarrassment (Tourangeau and Yan 2007). The priest does not wish to appear stigmatizing toward persons with MDs, particularly those with schizophrenia. Such a situation would lead the priest to identify the dangerousness in the perception of the others, as supported by the correlation between the subscales of perception of social distance by the others and of perception of dangerousness by the respondents $(r=0.30, p<0.0001 ; r=0.32, p<0.0001)$.

The results of this study also showed that compared to the younger priests, the older priests more firmly believed that persons with MDs have difficulties in affective relationships, are recognizable and kept at the distance by the others. These findings are in line with those from general population studies showing that negative attitudes and desire of social distance from persons with MDs are greater at older ages (Jorm and Oh 2009).

Priests considered persons with schizophrenia as less capable of participating in parish activities and sacraments than those with depression. It is likely that these differences may in part reflect the messages conveyed by the media contributing to social acceptance of persons with depression and to discrimination against those with schizophrenia (Magliano and Marassi 2018). Priests' reluctance regarding the involvement of persons with schizophrenia in religious activities may hinder connections of persons with these disorders with other churchgoers, further increasing the exclusion of these persons from contexts with potential rehabilitative effects. Furthermore, denying people with mental disorders access to pastoral care and the sacraments can adversely affect their mental and spiritual health. These findings highlight that persons with MDs, especially those with schizophrenia, are sometimes 
exposed to stigma even in religious settings. Educating priests about MDs and sensitizing them regarding the toxic effect of stigma (Magliano et al. 2016) on the lives and recovery processes of persons with MDs can be of dual benefit. On the one hand, it can facilitate the use of mental health services by believers with these disorders (Farrell and Goebert 2008), especially by those refusing to get medical help (Carey and Del Medico 2013). On the other hand, educating priests on stigma, can foster the fulfillment of the spiritual needs of these persons and their participation in the religious community of reference.

\section{Strengths and Limitations of the Study}

This is the first study carried out in Italy that specifically explored Catholic priests' views of persons with MDs and whether they varied in relation to a clinical description of schizophrenia or depression. Among the strengths of the study are the inclusion of a large sample randomly assigned to the diagnostic groups, the face-to-face data collection, and the high participation rate (92\%) suggesting that priests are interested in caring for persons with MD. The use of a self-reported questionnaire adapted to the study context and re-validated is an additional strength, also facilitating its replication in other religious contexts. The study has a number of limitations suggesting caution in the interpretation of its results. In particular, the inclusion of priests only from southern Italy, a geographical area where healthcare resources are poorer (Magliano et al. 2002) is a limitation that might have influenced the priests' beliefs about treatments and prognosis. The lack of other Italian studies of priests' beliefs about persons with MDs means we do not know whether our findings are generalizable to other areas of Italy. We are planning to extend the study to other geographical areas of Italy. Another limitation is the lack of a control group on priests' views of persons with a physical illness which may help in the interpretation of some results, particularly those related to the psychosocial consequences of MDs. This limitation prevents us from establishing how and whether priests' views of persons with MD are influenced by the stigma associated with these disorders. A further limitation is the lack of data on the participants' professional background, such as information on where the priests were trained and whether they had received formal training in mental health or psychology as part of their degree. These limitations prevent us from evaluating the potential influence of training on the priests' opinions. In addition, we did not collect data on priests' personal experience of MDs. Furthermore, the survey investigated only the opinions of priests, and may not reflect their actual behaviors with persons with MDs. However, the fact that almost half of the sample stated that persons with MD attended the own parish church to receive individual spiritual support and advices suggest that priests' opinions in part reflect the acceptance of these persons in the local religious community. Some of these limitations will be addressed in further studies at their planning stage.

Acknowledgements The authors thank: (a) Anna Corbisiero, Giuseppe Corbisiero, Vincenza Dell'Ungaro, Ilaria Esposito, Gaia Gaeta, Maria Grazia Iannotta, Fabiana Intoccia, Margherita Marrazzo, Antonietta Piscitelli, Sara Scia_psychology students at the time of the survey-for their contribution in the collection and input of the data; (b) the participating priests for giving us their time. 
Authors' Contribution Lorenza Magliano helped in conceptualization; Lorenza Magliano and Giulia Citarelli helped in methodology; Lorenza Magliano and Gaetana Affuso helped in statistical analysis; Lorenza Magliano and Gaetana Affuso contributed to writing — original draft preparation; Giulia Citarelli and Gaetana Affuso contributed to writing-review and editing; Lorenza Magliano helped in funding acquisition.

Funding Open access funding provided by Università degli Studi della Campania Luigi Vanvitelli within the CRUI-CARE Agreement. The study was supported by Grants from the University of Campania "Luigi Vanvitelli", Caserta, Italy (Approval No. 1, 24/01/2017 and No. 8, 23/06/2020).

Data Availability The data that support the findings of this study are available from the corresponding author upon reasonable request, which must include a protocol and statistical analysis plan and not be in conflict with our publication plan.

\section{Compliance with Ethical Standards}

Conflict of interest The authors declare that they have no conflict of interest.

Ethical Approval The study was approved by the Research Ethical Board of the Department of Psychology of the University of Campania "Luigi Vanvitelli" (Approval No. 22/2016 Department Board 6/12/16, and Approval No. 16/2019 Department Board 14/5/2019). The study was carried out in accordance with the principles of the Declaration of Helsinki.

Open Access This article is licensed under a Creative Commons Attribution 4.0 International License, which permits use, sharing, adaptation, distribution and reproduction in any medium or format, as long as you give appropriate credit to the original author(s) and the source, provide a link to the Creative Commons licence, and indicate if changes were made. The images or other third party material in this article are included in the article's Creative Commons licence, unless indicated otherwise in a credit line to the material. If material is not included in the article's Creative Commons licence and your intended use is not permitted by statutory regulation or exceeds the permitted use, you will need to obtain permission directly from the copyright holder. To view a copy of this licence, visit http://creativecommons.org/licen ses/by/4.0/.

\section{Appendix 1}

Some people sometimes seem unable to distinguish between things that really happen and are experienced by other people, and things that happen only in their mind. Sometimes, these people believe or say things that seem bizarre or absurd to other people, or hear voices, smell things, or see images that other people do not. Sometimes, these people may have difficulty expressing their feelings or behaving appropriately (for instance, they may cry in response to a positive event, or may appear happy following an unpleasant one), or they may remain shut up in their house for a long time, or talk very little or not at all. They behave as if they lived in a world of their own, apparently without interest in anything or anybody. Sometimes they may have muddled thoughts, may invent odd or incomprehensible words, may lose the thread of the speech, or they may jump from one issue to another with no apparent reason. 


\section{Appendix 2}

Some people sometimes feel sad, down, unable to feel pleasure, or to have interest for those activities they liked in the past. Sometimes, these people feel incompetent, may believe to be derided by the others, and make themselves feel guilty for trivial things. These people may have no hope for future and when their feelings of sadness and worthlessness become unbearable, they may decide to stop living. Sometimes, these people may have difficulties in eating and sleeping regularly, and may feel poor concentrated or physically tired. Other times, they may feel irritable and get annoyed with the others for unimportant things.

\section{References}

Aramouny, C., Kerbage, H., Richa, N., \& Rouhana, P. (2020). Knowledge, attitudes, and beliefs of catholic clerics' regarding mental health in Lebanon. Journal of Religion and Health, 59, 257-276. https ://doi.org/10.1007/s10943-019-00758-1.

Bonelli, R. M., \& Koenig, H. G. (2013). Mental disorders, religion and spirituality 1990 to 2010: A systematic evidence based review. Journal of Religion and Health, 52, 657-673. https://doi. org/10.1007/s10943-013-9691-4.

Carey, L. B., \& Del Medico, L. (2013). Chaplaincy and mental health care in Aotearoa New Zealand: An exploratory study. Journal of Religion and Health, 52, 46-65. https://doi.org/10.1007/s1094 3-012-9622-9.

Carpiniello, B. (2017). La lezione del Papa contro il pregiudizio: "Ho avuto bisogno dello psicanalista. Nessuna vergogna, fatevi aiutare. Retrieved July 24, 2020 from https://www.sanita24.ilsole24or e.com/art/medicina-e-ricerca/2017-09-04/la-lezione-papa-contro-pregiudizio-ho-avuto-bisognopsicanalista-nessuna-vergogna-fatevi-aiutare122314.php?uuid=AE4XmENC\&refresh_ce=1.

Curlin, F., Ryan, E. L., Marshall, S. O., Chin, H., Lantos, J. D., Koenig, H. G., et al. (2007). Religion, spirituality, and medicine: Psychiatrists' and other physicians' differing observations, interpretations, and clinical approaches. American Journal of Psychiatry, 164(12), 1825-1831. https://doi. org/10.1176/appi.ajp.2007.06122088.

Deacon, B. J. (2013). The biomedical model of mental disorder: A critical analysis of its validity, utility, and effects on psychotherapy research. Clinical Psychology Review, 33(7), 846-861. https://doi. org/10.1016/j.cpr.2012.09.007.

Delaney, H. D., Miller, W. R., \& Bisonó, A. M. (2007). Religiosity and spirituality among psychologists: A survey of clinician members of the American Psychological Association. Professional Psychology, 38(5), 538-546. https://doi.org/10.1037/0735-7028.38.5.538.

Ellison, C., Vaaler, M., Flannelly, K., \& Weaver, A. (2006). The clergy as a source of mental health assistance: What Americans believe. Review of Religious Research, 48(2), 190-211.

Eurobarometer. (2012). Discrimination in the EU in 2012, Special Eurobarometer 383, p. 233. Retrieved July 31, 2020 from https://web.archive.org/web/20121202023700/http://ec.europa.eu/public_opini on/archives/ebs/ebs_393_en.pdf.

Farrell, J., \& Goebert, D. A. (2008). Collaboration between psychiatrists and clergy in recognizing and treating serious mental illness. Psychiatric Services, 59(4), 437-440. https://doi.org/10.1176/ ps.2008.59.4.437.

Hackett, C. (2011). The global religious landscape. Retrieved July 24, 2020 from https://www.pewforum. org/2017/04/05/the-changing-global-religious-landscape/.

Heffernan, S., Neil, S., Thomas, Y., \& Wetherhead, S. (2016). Religion in the recovery journey of individuals with experience of Psychosis. Psychological, Social and Integrative Approaches, 8(4), 346356. https://doi.org/10.1080/17522439.2016.1172334.

Hohmann, A. A., \& Larson, D. B. (1993). Psychiatric factors predicting use of clergy. In E. L. Worthington Jr. (Ed.), Psychology and Christianity (Vol. 7, pp. 71-84)., Psychotherapy and religious values USA: Baker Book House.

IBM Corp. Released 2012. IBM SPSS Statistics for Windows, Version 21.0. Armonk, NY: IBM Corp. 
Ince, P., Haddock, G., \& Tai, S. (2016). A systematic review of the implementation of recommended psychological interventions for schizophrenia: Rates, barriers, and improvement strategies. Psychology and Psychotherapy, 89(3), 324-350. https://doi.org/10.1111/papt.12084.

Ipsos Game Changers. (2017). Retrieved July 24, 2020 from https://www.ipsos.com/en.

Jacobsson, L., \& Merdasa, F. (1991). Traditional perceptions and treatment of mental disorders in western Ethiopia before the 1974 revolution. Acta Psychiatrica Scandinavica, 84(5), 475-481. https:// doi.org/10.1111/j.1600-0447.1991.tb03180.x.

Jorm, A. F., \& Oh, E. (2009). Desire for social distance from people with mental disorders. Australian and New Zealand Journal of Psychiatry, 43(3), 183-200. https://doi.org/10.1080/000486708026533 49.

Jöreskog, K. G., \& Sörbom, D. (1996). LISREL 8: User's Reference Guide. USA: Scientific Software International.

Koenig, H. G. (2009). Research on religion, spirituality, and mental health: A review. Canadian Journal of Psychiatry, 54(5), 283-291.

Kvaale, E. P., Haslam, N., \& Gottdiener, W. H. (2013). The 'side effects' of medicalization: A metaanalytic review of how biogenetic explanations affect stigma. Clinical Psychology Review, 33(6), 782-794. https://doi.org/10.1016/j.cpr.2013.06.002.

Lafuze, J., Perkins, D. V., \& Avirapattu, G. A. (2002). Pastors' perceprion of mental disorders. Psychiatric Services, 53(7), 899-902. https://doi.org/10.1176/appi.ps.53.7.900.

Latkin, C. A., Edwards, C., Davey-Rothwell, M. A., \& Kobin, K. E. (2017). The relationship between social desirability bias and self-reports of health, substance use, and social network factors among urban substance users in Baltimore, Maryland. Addictive Behaviors, 73, 133-136. https:// doi.org/10.1016/j.addbeh.2017.05.005.

Leavey, G. (2010). The appreciation of the spiritual in mental illness: A qualitative study of beliefs among clergy in the UK. Transcultural Psychiatry, 4(47), 571-590. https://doi. org/10.1177/1363461510383200.

Linden, S. C., Harris, M., Whitaker, C., \& Healy, D. (2010). Religion and psychosis: The effects of the Welsh religious revival in 1904-1905. Psychological Medicine, 40(8), 1317-1323. https:// doi.org/10.1017/S0033291709991917.

Magliano, L., Marasco, C., Fiorillo, A., Malangone, C., Guarneri, M., \& Maj, M. (2002). Working group of the italian national study on families of persons with schizophrenia: The impact of professional and social network support on the burden of families of patients with schizophrenia in Italy. Acta Psychiatrica Scandinavica, 106(4), 291-298. https://doi.org/10.103 4/j.1600-0447.2002.02223.x.

Magliano, L., \& Marassi, R. (2018). "Schizophrenia" and "psychosis" in Italian national newspapers: Do these terms convey different messages? Schizophrenia Research, 197, 611-612. https://doi. org/10.1016/j.schres.2018.02.021.

Magliano, L., Petrillo, M., Ruggiero, G., \& Schioppa, G. (2018). Schizophrenia and psychosis: Does changing the label change the beliefs? Schizophrenia Research, 193, 482-483. https://doi. org/10.1016/j.schres.2017.08.007.

Magliano, L., Rinaldi, A., Costanzo, R., De Leo, R., Schioppa, G., Petrillo, M., et al. (2016). Improving psychology students' attitudes toward people with schizophrenia: A quasi-randomized controlled study. American Journal of Orthopsychiatry, 86(3), 253-264.

Magliano, L., Schioppa, G., Costanzo, R., Petrillo, M., \& Read, J. (2017a). The opinions of italian psychology students about people diagnosed with depression and schizophrenia: A comparative study. Journal of Psychosocial Rehabilitation and Mental Health, 4, 147-157. https://doi. org/10.1007/s40737-017-0087-8.

Magliano, L., Strino, A., Punzo, R., Acone, R., Affuso, G., \& Read, J. (2017b). Effects of the diagnostic label 'schizophrenia', actively used or passively accepted, on general practitioners' views of this disorder. International Journal of Psychiatry, 63(3), 224-234. https://doi.org/10.1177/00207 64017695353.

Milstein, G., Bruce, M., Kennedy, G. J. \& Flannelly, K. (2003). Clergy roles in psychiatric care: Elder patients' views. Presented at integrating research on spirituality and health and well-being into service delivery: A research conference. Natcher center, NH Campus, Bethesda, MD.

Milstein, G., Midlarsky, E., Link, B. G., \& Raue, P. J. (2000). Assessing problems with religious content: A comparison of rabbis and psychologists. Journal of Mental and Nervous Disease, 188, 608-615. https://doi.org/10.1097/00005053-200009000-00008. 
Mohr, S., Borras, L., Nolan, J., Gillieron, C., Brandt, P. Y., Eytan, A., et al. (2012). Spirituality and religion in outpatients with schizophrenia: A multi-site comparative study of Switzerland, Canada and the United States. International Journal of Psychiatry in Medicine, 44(1), 29-52. https:// doi.org/10.2190/PM.44.1.c.

Mosher, L., Gosden, R., \& Beder, S. (2013). Drug companies and "Schizophrenia". In J. Read, R. Bentall, L. Mosher, \& J. Dillon (Eds.), Models of madness psychological, social and biological approaches to psychosis (2nd ed., pp. 125-139). London: Routledge.

Munizza, C., Argentero, P. Munizza., Coppa, A., Tibaldi, G., Di Giannantonio, M., Rucci, P., et al. (2013). Public beliefs and attitudes towards depression in Italy: A national survey. Plos One. https://doi.org/10.1371/journal.pone.0063806m.

Read, J., Magliano, L., \& Beavan, V. (2013). Public beliefs about the causes of schizophrenia: Bad things happen and can drive you crazy. In J. Read, R. Bentall, L. Mosher, \& J. Dillon (Eds.), Models of madness psychological, social and biological approaches to psychosis (2nd ed., pp. 143-156). London: Routledge.

Russinova, Z., Wewiorski, N., \& Cash, D. (2002). Use of alternative health care practices by persons with serious mental illness: Perceived benefits. American Journal of Public Health, 92, 16001603. https://doi.org/10.2105/92.10.1600.

Tepper, L., Rogers, S. A., Coleman, E. M., \& Malony, H. N. (2001). The prevalence of religious coping among persons with persistent mental illness. Psychiatric Services, 52, 660-665. https://doi. org/10.1176/appi.ps.52.5.660.

Thornicroft, G., Brohan, E., Rose, D., Sartorius, N., Leese, M., \& INDIGO Study Group. (2009). Global pattern of experienced and anticipated discrimination against people with schizophrenia: A crosssectional survey. Lancet, 31, 408-415. https://doi.org/10.1016/S0140-6736(08)61817-6.

Tourangeau, R., \& Yan, T. (2007). Sensitive questions in surveys. Psychological Bulletin, 133(5), 859883. https://doi.org/10.1037/0033-2909.133.5.859.

Vasegh, S., Rosmarin, D. H., Koenig, H. G., Dew, R. E., \& Bonelli, R. M. (2012). Religious and spiritual factors in depression. Hidawi Publishing Corporation Depression Research and Treatment. https:// doi.org/10.1155/2012/298056.

Wang, P. S., Berglund, P. A., \& Kessler, R. C. (2003). Patterns and correlates of contacting clergy for mental disorders in the United States. Health Services Research, 38, 647-673. https://doi. org/10.1111/1475-6773.00138.

Weber, S. R., \& Pargament, K. J. (2014). The role of religion and spirituality in mental health. Current Opinions in Psychiatry, 27(5), 358-363. https://doi.org/10.1097/YCO.0000000000000080.

Yamada, A. M., Lee, K. K., Kim, M. A., Moine, M., \& Oh, H. (2018). Beliefs about etiology and treatment of mental illness. Journal of Religion and Health, 58, 870-880. https://doi.org/10.1007/s1094 3-018-0720.

Youssef, J., \& Deane, F. (2003). Arabic-speaking religious leaders' perceptions of the causes of mental illness and the use of medication for treatment. Australian and New Zealand Journal of Psychiatry, 47(11), 1041-1050. https://doi.org/10.1177/0004867413499076.

Publisher's Note Springer Nature remains neutral with regard to jurisdictional claims in published maps and institutional affiliations. 